\title{
Orphée sauvé ou De la magie selon Novalis
}

\section{Lionel Richard}

\section{(2) OpenEdition}

Journals

Édition électronique

URL : https://journals.openedition.org/rbnu/3180

DOI : $10.4000 /$ rbnu.3180

ISSN : 2679-6104

\section{Éditeur}

Bibliothèque nationale et universitaire de Strasbourg

\section{Édition imprimée}

Date de publication : 1 novembre 2011

Pagination : 20-29

ISSN : 2109-2761

\section{Référence électronique}

Lionel Richard, "Orphée sauvé ou De la magie selon Novalis », La Revue de la BNU [En ligne], 4 | 2011, mis en ligne le 01 novembre 2011, consulté le 08 août 2021. URL : http://journals.openedition.org/ rbnu/3180; DOI : https://doi.org/10.4000/rbnu.3180

\section{(c) (i) (2) (-)}

La Revue de la BNU est mise à disposition selon les termes de la Licence Creative Commons Attribution - Pas d'Utilisation Commerciale - Partage dans les Mêmes Conditions 4.0 International. 
Hef
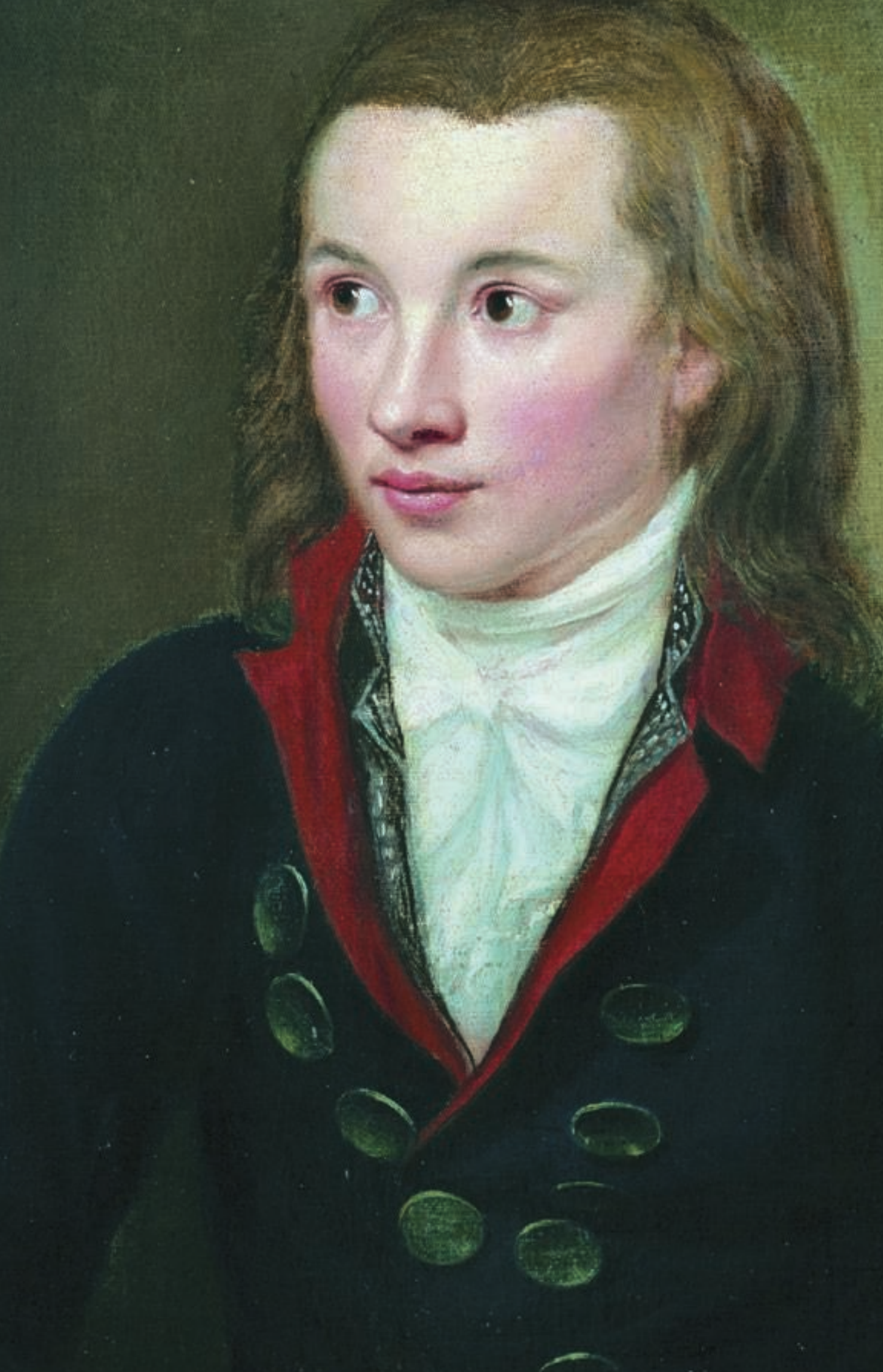

Portrait de Novalis (autour de 1799) par Franz Gareis (1775-1803 ; coll. Schloss Oberwiederstedt) 


\section{ORPHÉE SAUVÉ ou De la magie selon Novalis}

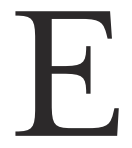

n 1798, dans l'Athenaeum, toute récente revue animée à Iéna par les frères August Wilhelm et Friedrich Schlegel avec l'objectif de poser les fondements du romantisme, paraît une suite d'aphorismes d'inspiration philosophique : Grains de pollen. Ces aphorismes, un peu dans la manière des "moralistes" français Vauvenargues et Chamfort, sont signés d'un nom inconnu jusque-là, Novalis'. Leur auteur est un ancien camarade d'études de Friedrich Schlegel à l'université de Leipzig, Friedrich von Hardenberg. Il a préféré publier sous un pseudonyme cette première sélection de ses " pensées mystiques ".

Originaire de Thuringe, fils aîné des dix enfants d'un baron que le Prince électeur de Saxe a placé à la tête de l'une de ses mines de sel, Novalis a été élevé dans l'austérité religieuse du piétisme. Enfance triste, dans un château du $13^{\mathrm{e}}$ siècle. Il connaît, à travers sa mère, puis ses frères ou sœurs, l'environnement pesant de la maladie et la mort. Après des études de droit, qu'il termine en 1794, il entre en stage auprès d'un bailli. Fin 1795, grâce à l'appui de son père, il est nommé assesseur adjoint à la direction des salines de Weissenfels. Intéressé par son travail, il aspire alors à mieux le maîtriser techniquement. Il s'oriente vers la profession d'ingénieur des mines et entreprend, de 1797 à la fin de 1799, des études scientifiques à l'Académie de Freiberg. Il y reçoit l'enseignement d'Abraham Gottlob Werner, l'un des géologues et minéralogistes les plus savants d'Europe, tout en étant fort controversé.

Entre-temps, il a été assailli par l'illumination de l'Amour et, rapidement, la perte de l'être aimé. Amené, lors d'une tournée de service, à rendre visite en novembre 1794 à un baron du voisinage, il entrevoit par une fenêtre du château le visage d'une jeune fille. Coup de foudre. Sophie von Kühn, enfant d'un premier lit de la baronne, n'a pas treize ans. Rencontre prédestinée Sophie ou l'incarnation de la Philosophie, l'amour de la Sagesse. Novalis l'idéalise. Elle devient, pour puérils que soient ses raisonnements et ses comportements, sa fiancée de rêve. Mais elle tombe malade. Elle meurt au bout de seize mois de souffrances. Il veut la suivre et ne s'y résout pas. Il se borne à se détacher spirituellement de la terre, en extase devant les splendeurs du royaume de la Nuit et de l'Éternité.

Surmontant peu à peu la douleur, transfigurant le personnage de Sophie en un Ange qui lui aurait été envoyé par le Ciel, il se lance dans une nouvelle vie, étudie les sciences naturelles, s'engage dans d'autres fiançailles à la fin de 1798. À partir de mars 1799, il transcende l'expérience de son deuil en un lyrisme mystique qui aboutit aux Hymnes à la nuit ${ }^{2}$, publiés au début de 1800 dans l'Athenaeum. Pour fuir l'isolement, il prête attention aussi aux débats littéraires et partage l'admiration de son ami Friedrich Schlegel pour le roman de Goethe désormais en librairie dans son intégralité, Les années d'apprentissage de Wilhelm Meister. Il le lit et le relit jusqu'à en analyser les procédés de narration, notant qu'y « alternent entretiens, descriptions et réflexions ". Soudain, retournement de son opinion sur ce roman et, en même temps, impulsion décisive pour élaborer une oeuvre personnelle sans concessions, en s'appuyant sur ses propres conceptions de la création poétique. Après avoir lié connaissance avec Ludwig Tieck et s'être passionné pour son roman qui relate les errances d'un élève d'Albert Dürer, Voyages de Franz Sternbald, l'évidence s’impose à lui : le Wilhelm Meister de Goethe est " anti- 
poétique au suprême degré quant à son esprit ». Comme il en informe Tieck, il souhaite écrire, en contrepartie, une " apothéose de la Poésie ».

En opposition au héros de Goethe, ancré dans le temps présent de la fin du $18^{\mathrm{e}}$ siècle, Novalis porte son choix sur un personnage historique du moyen-âge, Henri d'Ofterdingen. Il vise à définir, à travers lui, l'essence même du Poète, programme lui paraissant d'autant plus facilité que, paradoxalement, Ofterdingen est resté célèbre dans les mémoires sans que subsistent les traces d'aucune de ses œuvres. Pour identité, il n'a qu'une réputation transmise par la légende médiévale. Celle-ci a amplifié le peu d'éléments donnés à son sujet dans un recueil de sentences rimées $\mathrm{du} 13^{\mathrm{e}}$ siècle, en strophes dialoguées : La guerre des chanteurs au château de la Wartburg.

Initiateur d'un tournoi réunissant les ménestrels, vers l'an 1200 , à la cour du prince Hermann $1^{\text {er }}$ de Thuringe, Ofterdingen aurait été vaincu par l'un de ses pairs, Walter von der Vogelweide. Tandis que la mort lui était promise, la princesse, compatissante, aurait obtenu du jury que possibilité lui fût donnée de s'en remettre à un champion capable de le sauver. Il aurait proposé Klingsohr, renommé pour son expérience d'un voyage présumé en Nigromantie, le Royaume des Morts. Autorisé à gagner rapidement la Hongrie pour l'en ramener, il aurait eu la chance de voir son défenseur battre tous ses adversaires, et parmi eux celui tenu pour invincible, Wolfram von Eschenbach.

Barde sans œuvre attestée, simple écho d'une fiction, Ofterdingen est ainsi en mesure de représenter, aux yeux de Novalis, l'idée de Poésie que le prétendu destin d'Orphée a engendré mythiquement dans la culture occidentale. Comme Orphée sous la voix duquel chantaient les pierres, Novalis fait de son personnage un "élu". Il l'imagine portant en lui, fruit du hasard sous la sagesse de Dieu, une "disposition à la Poésie ". Mais pour devenir vraiment Poète après avoir pris conscience de cette révélation, Ofterdingen doit passer victorieusement par des épreuves indispensables : acquisition de la connaissance du Monde dans ce qu'il a de caché aux multitudes, assimilation intime et dépassement des interventions de l'Au-delà sur l'Icibas, initiation au mystère de la Vie dans son unité avec la Mort. Ensuite, il sera haussé à une Transfiguration. Il symbolisera un autre Orphée, signe sauvé de l'immortalité de la Poésie.

Jugeant le roman de Goethe une dégradation de la création poétique, Novalis construit l'itinéraire de son personnage à l'inverse de celui de Wilhelm Meister. Ce dernier, issu d'une famille bourgeoise de commerçants, découvre que ses talents artistiques étaient superficiels et renonce à une carrière théâtrale, à la séduction des effets d'illusion, pour se tourner vers une activité pratique dans la société. Ofterdingen, dont le père appartient à la basse noblesse tout en se livrant à un travail manuel d'artisan à la cour du souverain, donc en coïncidence parfaite avec les bases de la société féodale, se lancera sur la voie d'une existence éclairée par une quête spirituelle. Wilhelm est amené à s'épanouir intellectuellement dans le prolongement du rôle social émancipateur de la bourgeoisie, hors de la magie artificielle des feux de la rampe. Son rêve se brise sur la conscience progressive d'une obligation de réalisme. Ofterdingen verra sa destinée, au contraire, appelée par un rêve à s'échapper des réalités grossièrement visibles du Monde pour entrer en correspondance avec la magie des forces irradiantes, mais cachées, de l'univers.

Bien qu'il le situe intentionnellement au $13^{\mathrm{e}}$ siècle, avant le bouleversement de la Renaissance dans les croyances et connaissances, Novalis ne projette pas de composer un roman "historique", pas plus dans les événements que dans le décor. À travers Henri d'Ofterdingen, des allusions renvoient, certes, à des noms, des topographies, des épisodes repérables dans l'histoire médiévale de 1200 à 1250. Surviennent, entre autres, des croisés rentrés de Terre sainte avec une jeune captive. Mais l'arrière-pensée de Novalis est de polémiquer contre le type de "roman" qui domine à la fin du $18^{\mathrm{e}}$ siècle, celui d'un prolongement de l'épopée, cette fois en prose. Son roman à lui, il le veut "symbolique ", renouant avec les fonctions didactiques premières de l'épique.

Aussi ne retrace-t-il pas, à la Goethe, les cheminements d'un individu dans la formation qui doit le conduire à une profession. Son but est de formuler le modèle d'une " initiation ». À cette fin, néanmoins, il s'appuie volontairement, presque en parallèle, sur la charpente des Années d'apprentissage de Wilhelm Meister. Dans sa composition, c'est un roman qui lui semble en effet " intégralement ", tout hostile qu'il soit à son contenu, " un produit de l'art ". Par conséquent, la matrice du genre.

Comme le héros de Goethe, son personnage central est amené, peu après sa présentation, à voyager. Il est invité par sa mère à l'accompagner dans sa ville natale, Augsbourg, pour que son propre père voie enfin le petit-fils qu'il ne connaît toujours pas. Elle profite du déplacement de marchands pour leurs affaires. Qu'en 


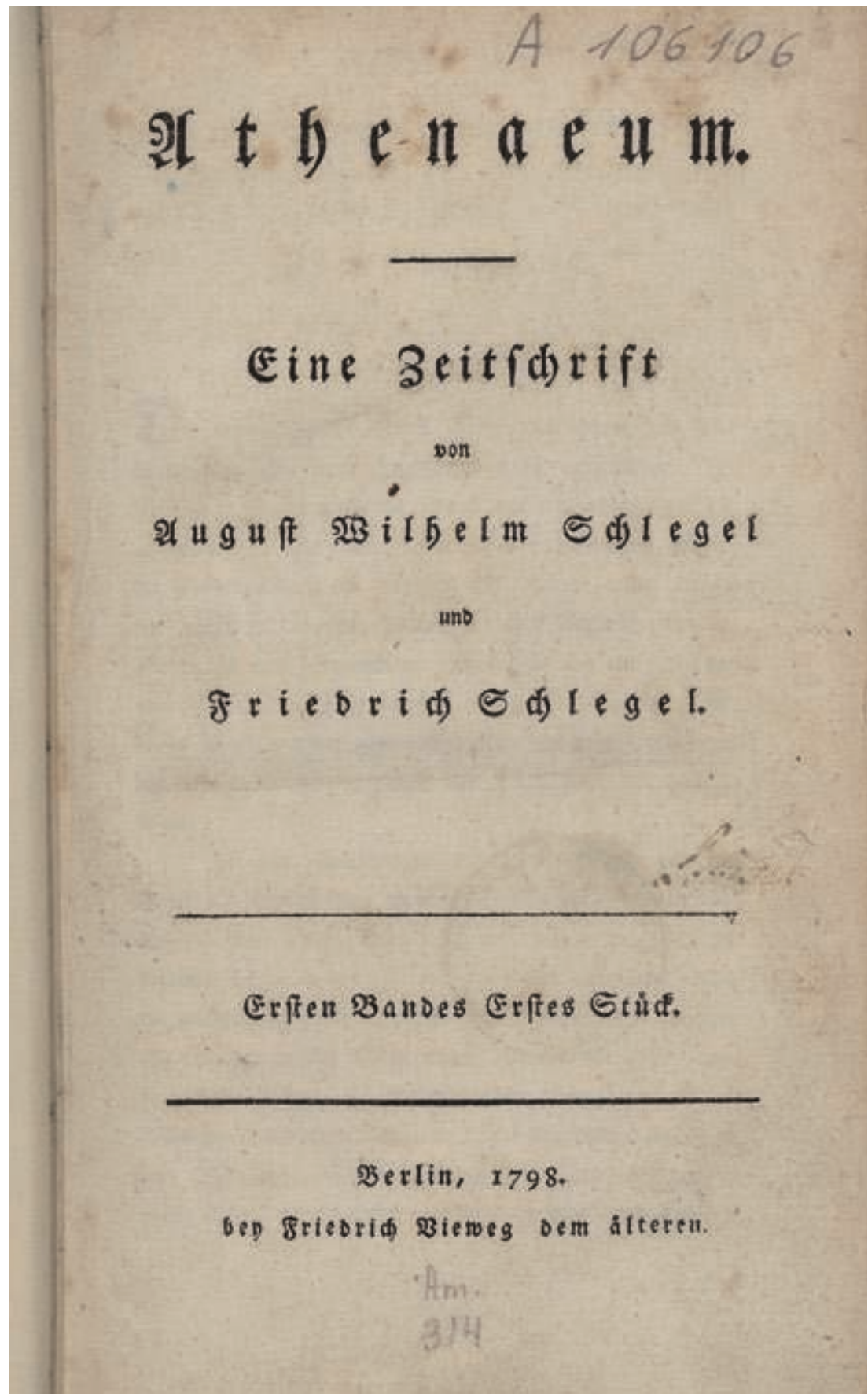

Le premier numéro de la revue Athenaeum (1798), où Novalis publia Grains de pollen (Blüthenstaub) (coll. BNU) 


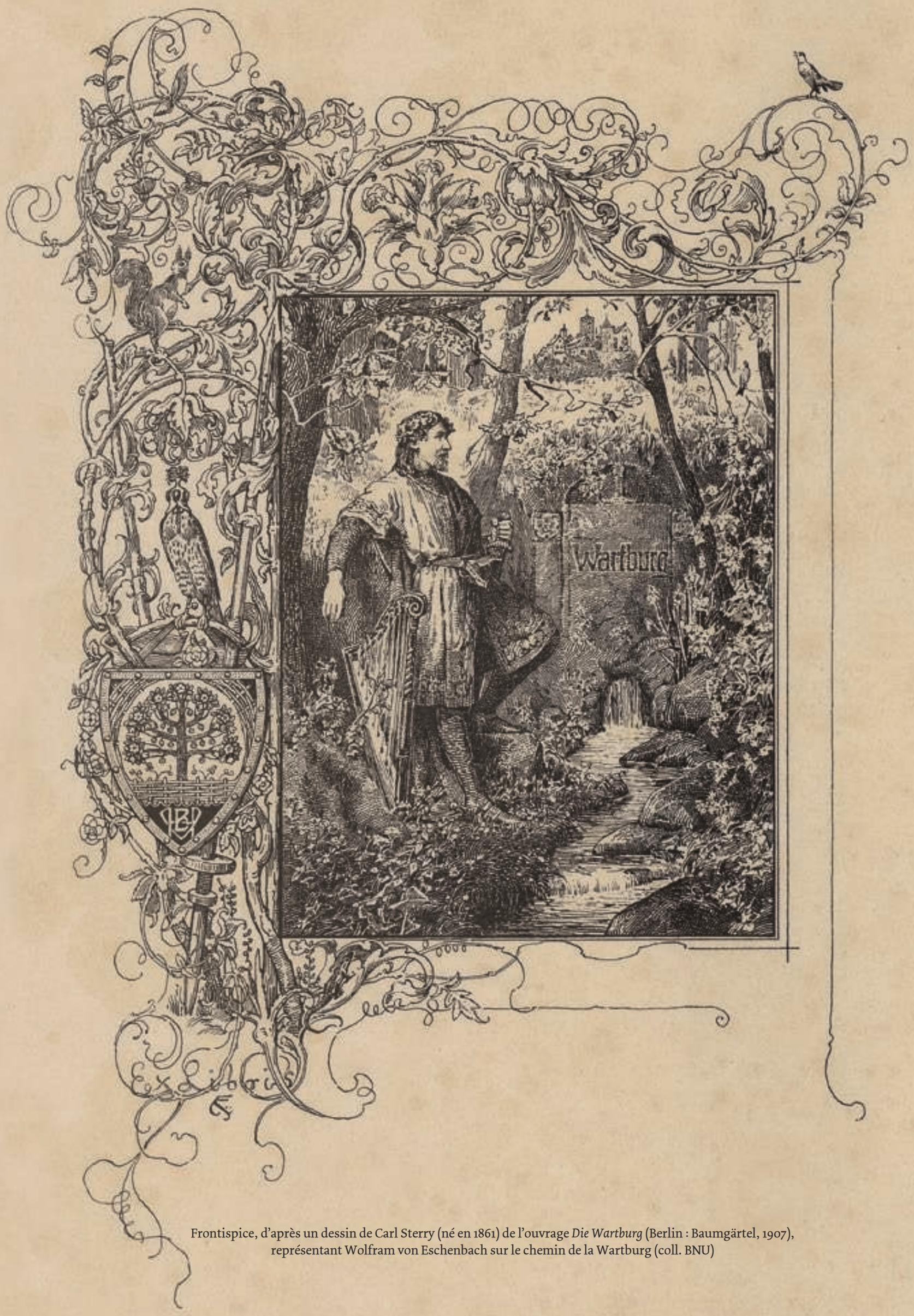


est-il d'Henri ? Né à Eisenach, au pied de la forteresse de la Wartburg, il n'est, à vingt ans, jamais sorti de sa ville. Sa formation intellectuelle est faible. Il l'a reçue par le biais de simples entretiens avec le chapelain de la cour. Pratiquement, il n'a lu aucun livre. S’il a entendu parler de la poésie, jamais il n'a eu de poèmes sous les yeux. C'est une " âme inexpérimentée ». D'où le titre que donne Novalis au premier volet de son roman : L'attente.

Ce roman s'ouvre sur l'attitude méditative d'Henri un soir dans son lit, attendant de s'endormir. Les récits d'un " étranger " rencontré peu avant suscitent en lui l'aspiration à découvrir une vague "Fleur bleue " que ce voyageur a évoquée. Succombant au sommeil, il se met à rêver. Rocs, torrents, forêt, falaise, couloir souterrain, et subitement il débouche sur une vaste salle éclairée, pourvue d'un bassin. Il s'y baigne. Afflux de sensations. Il se dirige au bord d'une source. Une Fleur bleue l'émerveille. Il s'en approche. Elle se métamorphose. De ses pétales transformés en une collerette bleue surgit " un tendre visage ». Malheureusement, voici Henri réveillé par sa mère qui l'appelle.

La première discussion matinale de la famille porte alors sur le phénomène du rêve. " Nous vivons à un âge du monde où le commerce direct avec le Ciel n'a plus lieu ", déclare le père. Autrement dit, l'intelligence rationnelle l'a emporté. Les annonces de miracles et prodiges ne sont plus crédibles. Henri, au contraire, estime que le rêve, sans être " une révélation d'en haut ", est un cadeau divin, et que le sien, en particulier, s'est introduit " dans son âme comme une grande roue qui lui impulse la force de son élan ".

Cet enthousiasme laisse le père dubitatif, mais son épouse lui rappelle qu'autrefois, juste avant qu'il ne la demande en mariage, il lui avait également raconté un rêve qu'il avait eu à Rome. Ce rêve, il s'en souvient en effet. Il le raconte. Lui aussi s'est retrouvé, guidé par un vieillard, dans une plaine où poussaient d'innombrables fleurs dont l'une, particulièrement merveilleuse, l'a plongé dans " un sentiment ineffable ". Était-elle bleue ? Il a oublié sa couleur. Ce qui s'est imposé à lui est l'image de sa future femme, tenant "dans ses bras un enfant resplendissant ".

Le rêve de la Fleur bleue, qui suggère un appel à pénétrer ailleurs, à se familiariser avec le mystère, et prophétise la révélation de l'Amour, est vécu par Henri comme le pressentiment d'un changement de destinée. Il est l'amorce d'un mouvement dans lequel le jeune homme a l'impression d'être entraîné. Mouvement circulaire, comme la roue, poussant à un départ qui ne développera qu'un retour vers soi-même.

Autant d'éléments symboliques donnés par Novalis, à l'entrée de son roman, pour en asseoir les bases. Rêver ouvre une porte sur la descente au fond de soi, "vers le dedans ", en établissant une relation avec les secrets de l'univers, et en invitant à s'interroger sur des événements déjà préfigurés. Passé, présent, futur se conjuguent dans la conscience en un flux indéterminé. Le Moi entre à l'unisson avec "l'âme " du Cosmos, de la Création divine, avec un faisceau de forces qui n'appartiennent pas au réel visible.

Le voyage d'Henri à Augsbourg vient enrichir concrètement le message qui lui a été communiqué d'une présence larvée de la Poésie tout autour de lui. Le chapelain a appris à son élève, selon ce que celui-ci raconte aux marchands, que les poèmes relevaient d'un " art noble ", très " répandu dans les temps anciens ". Les Poètes avaient été « honorés de la faveur divine, de sorte qu'enflammés par leur commerce avec l'invisible ils pouvaient proclamer sur terre, en des accents agréables, la Sagesse du Ciel ». Réponse des marchands : «Franchement, pour autant que nous prêtions l'oreille à leurs chants avec plaisir, jamais nous ne nous sommes préoccupés des secrets des Poètes. Il se peut qu'effectivement une entente toute particulière des astres entre eux commande à la venue au monde d'un Poète ; car avec cet art, à coup sûr, il en va d'une chose tenant véritablement du merveilleux ».

À la différence de la peinture et de la musique, expliquent encore les marchands, la Poésie ne tire pas ses éléments du "monde extérieur ". Elle ne copie pas le réel. C'est un " art secret ", pour lequel tout se passe " intérieurement ". Le Poète dispose du " savoir permettant d'éveiller en nous à son gré " les forces " secrètes " qui l'habitent. Il donne " à percevoir un magnifique monde inconnu " par le truchement des mots : "Comme de cavernes profondes s'élèvent en nous époques anciennes et futures, êtres humains innombrables, régions merveilleuses, avec les événements les plus étranges, et nous sommes arrachés au présent qui est connu de nous".

Les marchands sont des profanes dans l'art de la Poésie, mais ils en prodiguent à Henri une première connaissance, vulgaire et traditionnelle. Que lui indiquent-ils ? Qu'en deçà du "charme" opéré sur le public par l'œuvre chantée, son auteur a été obligé de s'approprier une haute compétence, tant dans l'expression adoptée que dans son contenu. Poète est celui qui, suite à sa pré- 
disposition intérieure, parvient à s'accorder avec l'harmonie naturelle de l'univers et qui, donc, a appris la " grammaire des correspondances universelles ", " les caractères internes et les vertus curatives des nombres, des plantes, de tous les êtres de la Création ".

Au fil de l'itinéraire d'Henri, la Poésie, clé des vérités du monde, va lui être rendue accessible par des médiateurs qui, à travers leurs récits d'histoires mythiques ou de leurs expériences, éventuellement à travers la déclamation de leurs poèmes ou l'exposé de leurs contes, la lui insufflent. Sans elle, pas d'Histoire. Sans elle, pas de Science. Elle est l'Un et le Tout. La foi du futur Poète dans la Création divine doit être absolue et déterminer sa volonté. À mesure des récits qu'il entend, Henri est amené à enrichir " son être intérieur déjà rempli d'intuitions ". Cependant, cette assimilation des nécessaires substances spirituelles ne suffit pas. Elle doit être complétée par l'acquisition d'une " linguistique " de l'enchantement. L'intermédiaire est ici Klingsohr, que présente à Henri son grand-père maternel d'Augsbourg, le vieux Schwaning, son " excellent ami ". Et à travers Klingsohr est offerte à Henri la révélation de l'Amour, impulsion capitale à l'inspiration, puisque le Poète est accompagné de sa fille, Mathilde, qui suscite aussitôt en lui des émotions jamais éprouvées, tout comme autrefois Sophie en Novalis lui-même.

Se contenter d'obéir à l'ivresse des sens ? Une telle voie, selon Klingsohr, serait bien loin d'être influente : "Ce que la Poésie demande en premier lieu, c'est à être exercée comme un art strict. Quand on la réduit à en tirer profit, elle cesse d'être poésie. Un Poète n'a pas à errer dans l'oisiveté toute la journée, courant après images et sentiments. Le chemin à prendre est l'inverse. Cœur pur et ouvert, aisance dans la réflexion et l'observation, habileté dans l'activité vivifiante mutuelle de toutes ses capacités, en ayant soin de l'entretenir, voilà les exigences de notre art ". Le succès, précise Klingsohr, provient de la qualité des moyens techniques. Le sujet du poème, lui, à condition de " reposer sur l'expérience vécue ", peut être le plus ordinaire qui soit. Choisir des thèmes éloignés, inconnus, ne manque pas de conduire à " un vide et pitoyable bruissement de mots, sans une étincelle de vraie Poésie ".

Quel est l'exercice de création poétique le plus élevé ? Pour Klingsohr, le " conte symbolique ". Ce genre ne saurait, par conséquent, être laissé aux néophytes sans compétence. Le Poète prouve par l'exemple, un peu plus tard, la validité de ce précepte, en exposant à la famille rassemblée autour du couple d'Henri et de Mathilde l'un des contes qu'il a écrits. En la circonstance, une parabole sur la réconciliation universelle dans la Poésie et l'Amour, synthétisant les mythes de toutes les religions.

Mais Henri d'Ofterdingen n'est que la fraction d'un ensemble qui, travaillé par Novalis de décembre 1799 à octobre 1800 , devait être composé de sept segments romanesques. Novalis avait en tête une encyclopédie philosophique, une somme contenant absolument tout de la connaissance du monde. C'est la mort qui l'a empêché d'achever son projet. Succombant à la tuberculose le 25 mars 1801, à 28 ans, il n'est parvenu à rédiger qu'une vingtaine de pages du deuxième segment prévu. Ses amis Tieck et Friedrich Schlegel ont, en juin 1802, publié séparément le premier segment. Ils lui ont intégré, en novembre 1802, le deuxième, pour former un seul et unique roman. "Sans une parfaite compréhension de soi-même, on n'apprendra jamais à vraiment comprendre les autres ", avait écrit Novalis dans Grains de pollen. Après avoir évalué tous les horizons du Cosmos, l'innocent jeune homme d'Eisenach devait donc redescendre au plus profond de son être, pour essayer d'élucider " le plus grand des mystères ", celui de soimême en sa "propre existence ". Dans la poursuite de son parcours spirituel, cette étape se borne, hélas, à son cheminement dans la montagne sous l'apparence d'un " pèlerin ", comme Novalis l'a maintenant baptisé. Ayant accédé à l'honneur de pouvoir se montrer Poète, il chante, en s'accompagnant d'un luth, son premier poème, où il exprime son amour de Mathilde et son aspiration à la retrouver. 


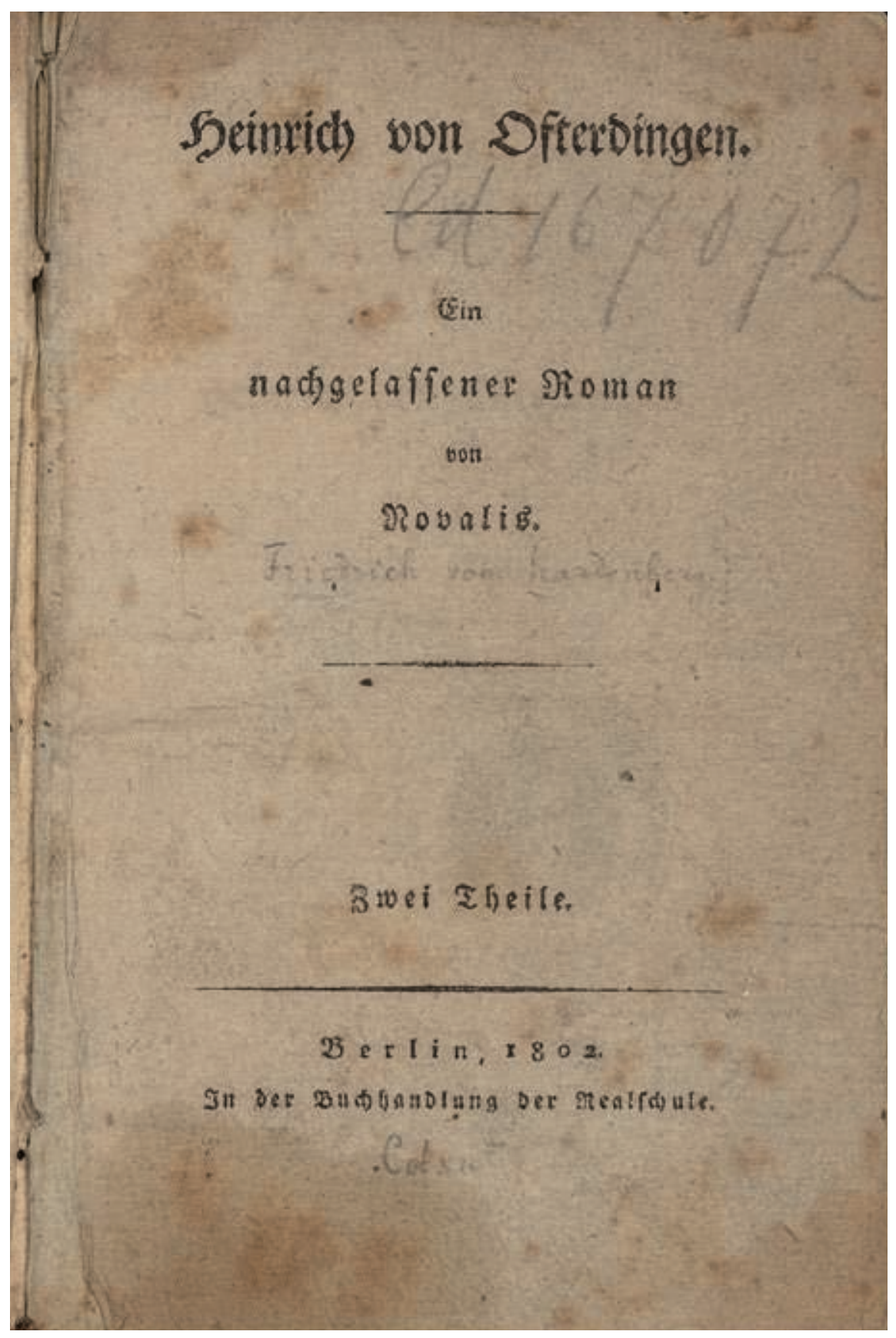

Edition originale d'Henri d'Ofterdingen (Berlin, 1802 ; coll. BNU) 
Selon un certain médecin Sylvestre qui se présente inopinément à lui, curieux redoublement d'un ermite avec lequel il s'est entretenu longuement à Augsbourg lors de son périple initial, "l'activité créatrice doit être désignée comme le fondement de toute existence ". Ayant été autrefois l'un des proches compagnons de son père, Sylvestre le met en garde sur ce qui est arrivé à celui-ci : " J'ai remarqué chez lui les signes annonciateurs d'un grand sculpteur... Mais le monde présent avait déjà poussé en lui des racines trop profondes dans son âme. Il n'a pas voulu prêter attention à l'appel de sa nature la plus intime, la rigueur sombre du ciel de sa patrie avait flétri en lui les tendres pousses des plantes les plus nobles, il est devenu un habile artisan, et l'enthousiasme n'a plus été pour lui que folie ".

Comment procéder pour libérer les capacités de création qui stagnent au fond de soi ? L'Amour est l'instigateur. Tout comme Orphée a perdu Eurydice, piquée par un serpent, Henri a été dépossédé de Mathilde. Il a été averti de sa disparition en un rêve prémonitoire : elle se baignait dans une eau profonde et s'est noyée. À l'instar d'Orphée gagnant les Enfers pour en ramener Eurydice, il lui faut, en vue de ressusciter Mathilde, pénétrer le Royaume des Morts.

Cette aventure aurait conduit Henri, si Novalis, d'après ses notes, avait achevéle deuxième segment de sa somme romanesque, à une grotte où Mathilde reposait endormie. Par l'entremise d'une étrange fillette, assise près du cercueil, il la tirait de sa léthargie. Le Poète Henri d'Ofterdingen atteignait alors un but ancien et chimérique, surhumain, dans lequel Orphée, trop impatient, avait échoué : il sauvait la condition humaine du tragique de ses limites. La présence en lui de la Poésie manifestait sa toute-puissance par cet acte démiurgique. L'Humanité pouvait s'en retourner à l'Âge d'or.

Que devait comprendre Henri à l'issue de ses pérégrinations ? Pour Novalis, le sens même de la Poésie et la nécessité de restaurer partout la Création poétique dans son règne. Celle-ci, a-t-il voulu montrer, n'est pas ce qui vient arracher temporairement le philistin à sa plate existence. Elle n'est pas distraction. C'est un absolu, avec le feu central qui lui correspond. Entretenant un lien souterrain avec l'esprit de la Création originelle, la Poésie est la seule apte à étouffer la trivialité des relations sociales sans idéal ni foi. C'est en ce sens qu'elle est "magie", source de merveilleux.

Le Poète n'est pas l'expert en artifice qu'est le magicien, mais un Mage, possédé d'une mission supérieure. Il exerce, grâce aux techniques du rythme et des sonorités qu'il a parfaitement incorporées, un pouvoir d'incantation et d'enchantement. Hors de toute religion instituée, il est le prêtre d'une régénération de l'Humanité, régénération fondée sur les forces créatrices actives d'Éros.

Novalis, théoricien de "l'idéalisme magique " ? S'il utilise effectivement cette notion, c'est pour qualifier des conceptions esthétiques se conjuguant à une philosophie, non pour exalter les divinations des Sages de l'Occultisme. En 1895, l'écrivain symboliste Maurice Maeterlinck ${ }^{3}$, dans son introduction à la traduction qu'il propose des Disciples à Saïs, œuvre rédigée sept ans avant Henri d'Ofterdingen, relativise les influences que Novalis a pu subir à travers ses lectures de philosophes comme Johann Gottlieb Fichte, François Hemsterhuis, Friedrich Wilhelm Schelling, ou sa découverte d'ouvrages " de théosophie, de théurgie, de pneumatologie transcendante, de cosmologie métaphysique ". Les cantiques du théologien suisse Gaspard Lavater, maître à penser de toutes les familles piétistes comme la sienne, puis la théosophie du cordonnier Jacob Böhme, dans laquelle Friedrich Schlegel l'a fortement incité à se plonger, ontils joué un rôle dans son orientation intellectuelle ?... Maeterlinck juge que non. Novalis n'était pas, estime$\mathrm{t}$-il, un esprit dogmatique. C'était « un mystique presque inconscient ", qui possédait en lui le « sentiment de l'unité secrète de l'univers » et communiquait spontanément avec "l'infini».

Cette vision répond à l'image qu'ont gardée de lui ceux qui l'ont connu d'assez près. Ils ont témoigné qu'il vivait tout naturellement dans la " région lumineuse " d'une "patrie originelle et pure". Ce sont les mots qu'emploie, dans ses souvenirs, le philosophe Henrich Steffens, qui eut l'occasion de le rencontrer à diverses reprises. Novalis, pour approfondir son expérience mystique, ce " chemin mystérieux " qui " mène vers l'intérieur ", selon la définition qu'il donne dans un aphorisme de ses Grains de pollen, n'avait pas besoin, semble-t-il, de s'engloutir dans les traités de Böhme ou autres Illuminés.

De toute manière, le lecteur " fait d'un livre, à vrai dire, ce qu'il veut ", considère l'auteur d'Henri d'Ofterdingen dans l'une de ses notes. L'acte de lecture est une " libre opération " et " personne ne peut me prescrire ce que je dois lire, ni comment ». Parmi tous ses papiers, il l'admet en 1797 auprès de Friedrich Schlegel ${ }^{4}$, " beaucoup de théosophie et d'alchimie ". La raison en est que l'une et l'autre, dans leur " masse bizarre et grotesque ", dans leur " fatras ", offrent un inépuisable réservoir de symboles qui stimulent l'imagination. 
Klingsohr l'a expliqué à Henri, l'origine de l'expression par la Poésie n'est pas un problème d'influences ou non: "Que la création poétique porte un nom particulier et que ses auteurs forment une corporation particulière est vraiment fâcheux. Absolument rien là de particulier. C'est le mode d'activité propre à l'esprit humain ". Reste à savoir échapper, pour ne pas laisser dévoyer cette faculté naturelle, à la banalisation et aux passions médiocres qui s'appliquent à rabaisser l'Humanité.

Avec châteaux, forêts, grottes, ermites et autres intermédiaires prétendus de l'Occulte, Henri d'Ofterdingen accumule tous les poncifs romantiques et leur attirail annexe. Pourtant, voilà qui n'a pas empêché son terreau de fructifier. Dans leur recours aux idées de Novalis, les symbolistes en ont même abouti à un excès de zèles. Formés en leur giron, André Breton et ses compagnons surréalistes les ont suivis plus modestement. L'initiation du jeune Henri à la Poésie leur a ouvert des portes pour tenter, à leur tour, dans la collusion du Rêve, de l'Amour, du Merveilleux, de débusquer de ses positions de force le "prosaïsme" quotidien universel. Vingt ans après la flambée du mouvement surréaliste, Paul Éluard ${ }^{6}$ proclame encore : « Les plus grandes merveilles passeront dans le langage commun ».

\section{Notes}

1 - Novalis, Schriften, herausgegeben von Paul Kluckhohn und Richard Samuel, 4 volumes, Stuttgart, W. Kohlhammer, 1960 et suiv.

2 - Cf. Novalis, Journal intime, suivi des Hymnes à la nuit et de fragments inédits, traduit de l'allemand par G. Claretie et S. Joachim-Chaigneau, introduction de Germaine Claretie, Paris, Stock/Delamain et Boutelleau, 1927

3 - Les disciples à Saï et les fragments de Novalis, traduits de l'allemand et précédés d'une introduction par Maurice Maeterlinck, Bruxelles, P. Lacomblez, 1895

4- Lettre du 26 décembre 1797, cf. Friedrich Schlegel und Novalis, Biographie einer Romantikerfreundschaft in ihren Briefen [Januar 1792-Juli 180o], herausgegeben von Max Preitz, Darmstadt, H. Gentner, 1957

5 - Cf. Novalis, Henri d'Ofterdingen, traduit et annoté par Georges Polti et Paul Morisse, préface d'Henri Albert, Paris, Mercure de France, 1908

6 - Aujourd'hui la poésie, conférence à l'Institut français de Prague, 9 avril 1946, in Paul Éluard, Le poète et son ombre, textes inédits présentés et annotés par Robert D. Valette, Paris, Seghers, 1963, p. 109

\section{Lionel Richard}

